\title{
Mathematical Modelling and Dynamic Analysis of an Offshore Drilling Riser
}

\author{
Maolin Liao, ${ }^{1}$ Gaowei Wang, ${ }^{1}$ Zhiying Gao, ${ }^{1}$ Yipeng Zhao, ${ }^{2}$ and Ruifeng $\mathrm{Li}^{3}$ \\ ${ }^{1}$ School of Mechanical Engineering, University of Science and Technology Beijing, Beijing, China \\ ${ }^{2}$ CNPC Engineering Technology R\&D Company Limited, Beijing, China \\ ${ }^{3}$ Tianjin Branch of CNOOC Company Limited, Tianjian, China \\ Correspondence should be addressed to Maolin Liao; liaomaolin@ustb.edu.cn
}

Received 10 July 2020; Revised 19 August 2020; Accepted 20 August 2020; Published 29 August 2020

Academic Editor: Qilong Xue

Copyright ( $\odot 2020$ Maolin Liao et al. This is an open access article distributed under the Creative Commons Attribution License, which permits unrestricted use, distribution, and reproduction in any medium, provided the original work is properly cited.

In this paper, a dynamic model of an offshore drilling riser is developed based on the Hamilton principle. The developed dynamic model is transformed into a finite element model by introducing an approximate solution which chooses the Hermite cubic interpolation function of bending beam element as the shape function. Thereafter, the standard Newmark integration is applied to numerically simulate the dynamic responses of offshore drilling risers with varied system parameters, including the length of riser, top tension ratio, and buoyant factor. Based on the results of numerical simulation, under the influences of sea wind, sea current, and the periodic excitation of sea wave, the offshore drilling riser experiences a fast lateral deflection phase in the beginning, a reciprocating deflection phase in the following long duration, and then, a periodic oscillation when it reaches the dynamic stable condition, respectively. The riser system working in deeper water with a higher top tension ratio and a lower buoyant factor shows more controllable vibration and less lateral deflection.

\section{Introduction}

The exploitation of offshore oil and gas resources faces complex ocean climate and environment; hence, the requirements for the reliability of equipment and the safety of operation are of high standard. Offshore drilling is different from onshore drilling since drilling rigs and drilling mud reach the wellhead through a drilling riser system whose length is determined by sea depth. The riser system can be viewed as an extension of borehole since its bottom side joins with a blowout preventer which is installed on the wellhead; while its topside is connected with a drilling platform or a drilling vessel via a telescopic joint and flexible joint. During the offshore drilling, the riser swings along with the ocean environmental loads, which will make it deflect and vibrate. Both the excessive deflection and the severe vibration will challenge the stability of the riser and, further, bring the potential risk for the offshore drilling operation.

The investigations of the dynamics of offshore drilling risers are traced back to the 1970s. According to both the fourth-order differential equation of the Euler-Bernoulli beam and the Morison equation, Burke [1] built up a dynamic model of a riser system applied in $800 \mathrm{ft}$ sea depth according to Newtonian mechanics. Based on Burke's work, scholars applied the finite element method to analyse the riser dynamics for two-dimension $[2,3]$ or three-dimension conditions [4, 5]. Thereafter, rather than using the Euler-Bernoulli beam, Yazdchi and Crisfeld [6] applied the Reissner-Simo beam to develop the mathematical model of the riser system to enhance the analysis of shear deformation of the riser. Instead of using the beam models, Nair and Baddour [7] divided the riser system into a number of pieces which were connected by a series of spring-damper units, which can also be viewed as another type of the finite element method. In the recent years, both Yang et al. [8] and Wang et al. [9] considered the interactions of coupled factors during the dynamic analysis of a riser system, so that global dynamics of the riser can be obtained. Mao et al. $[10,11]$ designed an experimental system to measure the dynamic responses of the riser under shear flow with vortex-induced 
vibration. Wu et al. [12] developed a dynamic model for offshore drilling riser based on the Hamilton principle and conducted the nonlinear vibration analysis of riser, and their results illustrated that both periodic motions and chaotic scenarios can be observed during vibration of the riser. In addition, rather than applying empirical hydrodynamic coefficients of a flexible pipe, Ren et al. [13] carried out experiments to identify both the drag coefficient and added mass coefficient which are generally used in the Morison equation to determine the distribution of ocean loads applied on the riser. Liu et al. [14] employed the Forgetting Factor Least Squares method for the identification of timevarying hydrodynamics of a flexible cylinder under modulated vortex-induced vibration.

Based on the dynamic analysis of riser, the control of vibration and deflection of the riser are further considered by a number of authors. Chang et al. [15] analysed both the environmental factors and the operational factors to optimize an offshore drilling riser. Ge et al. [16] added the reverse actuator at the topside of the riser system to restrict its lateral deflection. Aiming at minimizing the rotation angle of the lower flexible joint, Wang et al. [17] focused on the optimization of the top tension of the riser. Based on the dynamic model developed by Abaqus, Liao et al. [18] applied Isight to carry out the multiobjective optimization design of an offshore drilling tube system with a pipe-in-pipe structure. Guo et al. [19] considered the vibration suppression of a variable length drilling riser system. Liu et al. [20] proposed an optimization method for designing the suppression device configuration of risers, which can slow down the fatigue damage of risers caused by vortex-induced vibration. In addition, $\mathrm{Wu}$ et al. proposed both an adaptive output feedback control method [21] and an observer-based nonlinear control method [22] to deal with the issues when structures suffered unmodeled dynamics or parametric uncertainties. Such two methods are expected to be also feasible for analysis of riser dynamics since the ocean environmental loads are unavailable in most cases in realworld applications.

According to the aforementioned works, the dynamic characteristics of offshore drilling risers have been continuously investigated during the past half century; however, in the published papers, several important steps both in the process of the dynamic modelling and in the solution procedure via finite element analysis were always omitted. Hence, in this paper, a completed procedure about both the model development and the corresponding solution flow path for offshore drilling riser is intended to be introduced in detail, which is also a foundation work for the authors' further research plan to analyse both the geometric nonlinearity and the contact nonlinearity on the dynamic responses of a riser-well-drilling string coupling system when it is applied in deep water drilling.

The rest of this paper is organized as follows. Section 2 introduces the mathematical modelling of an offshore drilling riser. Then, the developed dynamical model is transformed into a finite element model, which is, then, solved by the Newmark integration in Section 3. Subsequently, the dynamic characteristics of the riser system are discussed, and the influences of three key system parameters on the dynamic responses of the riser are compared in Section 4. At last, concluding remarks are provided in Section 5 .

\section{Mathematical Modelling of an Offshore Drilling Riser}

In this section, the Hamilton principle will be applied to develop the dynamic model of an offshore drilling riser. The physical model of a riser element under lateral deflection is shown in Figure 1, based on which the kinetic energy, potential energy, and external work of a riser element can be described mathematically, and thus, an entire model of the offshore drilling riser can be developed.

2.1. Kinetic Energy. Without considering the influence of drill string, the kinetic energy of an offshore drilling riser system includes both the vibration of the riser and the motion of the inside drilling mud, which can be expressed as

$$
T(x, t)=\frac{1}{2} \int_{0}^{L}\left(m_{r}+m_{m}\right)\left(\frac{\partial \varpi(x, t)}{\partial t}\right)^{2} \mathrm{~d} x,
$$

where $₫(x, t)$ is the lateral deflection of the riser segment, and the mass for a riser segment with unit length $\left(m_{r}\right)$ and the mass of the drilling mud inside this riser segment $\left(m_{m}\right)$ can be calculated as

$$
\begin{aligned}
& m_{r}=\frac{\pi}{4}\left(D_{r}^{2}-d_{r}^{2}\right) \rho_{r}, \\
& m_{m}=\frac{\pi}{4} d_{r}^{2} \rho_{m},
\end{aligned}
$$

where $D_{r}$ and $d_{r}$ are the external diameter and the internal diameter of the riser, respectively; $\rho_{r}$ and $\rho_{m}$ are the density of riser and that of drilling mud.

2.2. Potential Energy. According to the theorem of strain energy, the bending deformation energy can be expressed as

$$
V_{b}(x, t)=\frac{M(x, t)^{2}}{2 E I_{x}}
$$

where the bending moment can be calculated as

$$
M(x, t)=-E I_{x} \frac{\partial^{2} \varpi(x, t)}{\partial x^{2}} .
$$

Hence, the strain energy due to the lateral bending deformation of a riser segment is

$$
V_{b}(x, t)=\frac{1}{2} E I_{x}\left(\frac{\partial^{2} \varpi(x, t)}{\partial x^{2}}\right)^{2},
$$

where $E$ is the elastic modulus of the riser, and the inertia moment of the cross section to the central axis of the riser is

$$
I_{x}=\frac{\pi}{64}\left(D_{r}^{4}-d_{r}^{4}\right)
$$




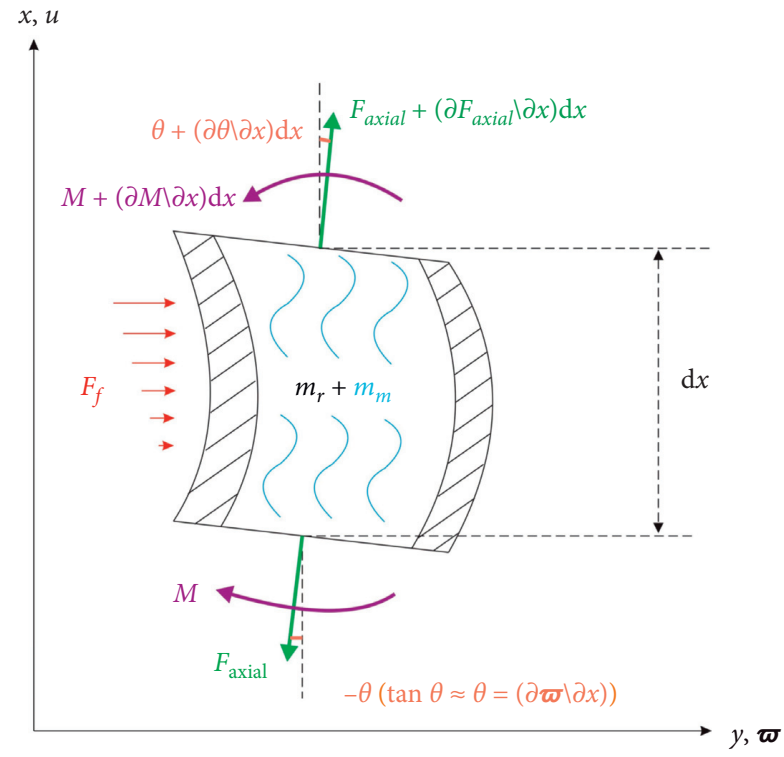

Figure 1: Physical model of an offshore drilling riser element under lateral deflection.

On the other hand, as an internal force of the riser, the axial tension varies as the axial position of the riser, and it is determined by both the top tension and the buoyant weight of the riser with drilling mud, which can be calculated as

$$
\begin{aligned}
F_{\text {axial }}(x)= & R_{\text {top }}\left(m_{r}+m_{m}\right) g L \\
& -\int_{x}^{L}\left(1-f_{b}\right)\left(m_{r}+m_{m}\right) g \mathrm{~d} x,
\end{aligned}
$$

where $L$ is the total length of the riser, $f_{b}$ is the buoyant factor, and $R_{\text {top }}$ is the ratio between the top tension and the weight of the riser with drilling mud.

Due to the bending deformation of the riser, the axial tension will work on the lateral direction, and by considering the simplification of a small deflection angle $(\theta)$, as shown in Figure 1, the work for a riser segment can be expressed as

$$
\begin{aligned}
V_{T}(x, t) & =\int\left(F_{\text {axial }}(x) \cdot \frac{\partial \varpi(x, t)}{\partial x}\right) \mathrm{d} \frac{\partial \varpi(x, t)}{\partial x} \\
& =\frac{1}{2} F_{\text {axial }}(x)\left(\frac{\partial \varpi(x, t)}{\partial x}\right)^{2} .
\end{aligned}
$$

Hence, the total potential energy for a riser segment is

$$
\begin{aligned}
V(x, t) & =V_{b}(x, t)+V_{T}(x, t), \\
& =\frac{1}{2} E I_{x}\left(\frac{\partial^{2} \varpi(x, t)}{\partial x^{2}}\right)^{2}+\frac{1}{2} F_{\text {axial }}(x)\left(\frac{\partial \Phi(x, t)}{\partial x}\right)^{2} .
\end{aligned}
$$

2.3. External Work. For an offshore drilling riser system, the external work comes from ocean environmental loads $\left(F_{f}\right)$, which can be calculated as

$$
W(x, t)=F_{f}(x, t) \cdot \omega(x, t) .
$$

According to Morrison's equation [1], the ocean environmental loads acting on the riser include both the drag force $F_{D}$ and the inertial force $F_{I}$, which can be calculated as

$$
\begin{aligned}
F_{f}(x, t)= & F_{D}(x, t)+F_{I}(x, t), \\
F_{D}(x, t)= & \frac{1}{2} C_{d} D \rho_{w}\left(u_{w}(x, t)+u_{c}(x)-\frac{\partial \varpi(x, t)}{\partial t}\right) \\
& \cdot\left|u_{w}(x, t)+u_{c}(x)-\frac{\partial \varpi(x, t)}{\partial t}\right|, \\
F_{I}(x, t)= & \frac{1}{4} \pi D^{2} \rho_{w}\left(C_{i} a_{w}(x, t)-\left(C_{i}-1\right) \frac{d^{2} \varpi(x, t)}{d t^{2}}\right),
\end{aligned}
$$

where $C_{d}, C_{i}$, and $\rho_{w}$ are the drag coefficient, inertial coefficient, and sea-water density, respectively. Moreover, in order to solve the being developed model numerically later on, a linear factor $B$ is introduced to replace $\mid u_{w}+u_{c}-$ $(\partial \varpi / \partial t) \mid$ in equation (14) as follows [12]:

$$
B(x, t)=\sqrt{\frac{8}{\pi} \cdot \frac{1}{T_{w}} \int_{0}^{T_{w}}\left(u_{w}(x, t) u_{c}(x)-\frac{\partial \omega(x, t)}{\partial t}\right)^{2} \mathrm{~d} t .}
$$

In addition, Airy wave theory [23] can be applied to obtain the velocity and the acceleration of sea wave as

$$
\begin{aligned}
& u_{w}(x, t)=\frac{\pi h_{w}}{T_{w} e^{2 \pi\left((x-L) / L_{w}\right)} \cos \left(2 \pi / T_{w} t\right)}, \\
& a_{w}(x, t)=\frac{2 \pi^{2} h_{w}}{T_{w}^{2} e^{2\left(\pi(x-L) / L_{w}\right)} \sin \left(2 \pi / T_{w} t\right)},
\end{aligned}
$$

where $h_{w}$ and $T_{w}$ are the amplitude and period of the sea wave, respectively; thus, the length of sea wave can also be calculated as

$$
L_{w}=\frac{g T_{w}^{2}}{2 \pi} .
$$

Meanwhile, Ekman drift theory [23] can be applied to calculate the flow velocity of sea current as

$$
\begin{aligned}
& u_{c}(x)= \begin{cases}v_{w c} \cdot \frac{\left(x-L+H_{c}\right)^{1 / 7}}{H_{c}+v_{d} \cdot(x-L)}, & x \leq H_{c}, \\
v_{d}(x-L)^{1 / 7}, & x>H_{c},\end{cases} \\
& H_{c}=\frac{7.6 v_{w}}{\sqrt{\sin \varphi}}, \\
& v_{w c}=K_{w} v_{w}, \\
& v_{d}=\frac{R_{d}}{R_{M}} v_{t},
\end{aligned}
$$


where $H_{c}$ is the friction depth, $\varphi$ is the geographic latitude, $v_{w}$ is the wind speed on the marine surface, $v_{w c}$ is the velocity of wind current, $K_{w}$ is the factor of wind speed, $v_{d}$ is the flow velocity of tide, $v_{t}$ is the flow velocity of sea surface tide, and $R_{M}$ is the strong tidal range, while $R_{d}$ is the normal tidal range.

2.4. Dynamic Model of an Offshore Drilling Riser. Based on the abovementioned analyses, the Hamilton principle is applied to develop the dynamic model of an offshore drilling riser, and the total energy of the entire offshore drilling riser system in a period of sea wave can be described as

$$
\prod_{P}=\int_{0}^{T_{w}} \int_{0}^{L}(T(x, t)-V(x, t)+W(x, t)) \mathrm{d} x \mathrm{~d} t,
$$

where $\prod_{P}$ is the functional equation of the skew curve $\omega(x, t)$, whose variation can be expanded as

$$
\begin{aligned}
\delta \prod_{P} & =\int_{0}^{T_{w}} \int_{0}^{L}(\delta T(x, t)-\delta V(x, t)+\delta W(x, t)) \mathrm{d} x \mathrm{~d} t \\
& =\int_{0}^{T_{w}} \int_{0}^{L} \delta\left(\begin{array}{c}
\left(\frac{1}{2}\left(m_{r}+m_{m}\right)\left(\frac{\partial \varpi(x, t)}{\partial t}\right)^{2}\right)-\delta\left(\frac{1}{2} E I_{x}\left(\frac{\partial^{2} \Phi(x, t)}{\partial x^{2}}\right)\right)^{2} \\
+\frac{1}{2} F_{\text {axial }}(x)\left(\frac{\partial \omega(x, t)}{\partial x}\right)^{2}+\delta\left(F_{f}(x, t) \cdot \omega(x, t)\right)
\end{array}\right) \mathrm{d} x \mathrm{~d} t,
\end{aligned}
$$

$=0$.

In addition, the top side of the riser system drifts with the drilling platform or vessel since they are connected via a flexible joint whose rotational stiffness is $K_{t}$, while the bottom side of the riser is fixed at the wellhead where another flexible joint is used, and its rotational stiffness is $K_{b}$. Therefore, the boundary condition for the top side is

$$
\left\{\begin{array}{l}
\omega(L, t)=C_{\text {drifting, }} \\
E I_{x} \frac{\partial^{2} \omega(L, t)}{\partial x^{2}}=K_{t} \frac{\partial \omega(L, t)}{\partial x} .
\end{array}\right.
$$

Similarly, the boundary condition for the bottom side is

$$
\left\{\begin{array}{l}
\bowtie(0, t)=0, \\
E I_{x} \frac{\partial^{2} \oplus(0, t)}{\partial x^{2}}=K_{b} \frac{\partial \omega(0, t)}{\partial x} .
\end{array}\right.
$$

Comparing with the boundary conditions, the initial conditions for the riser system are quite simple. In the beginning of numerical simulation, all the displacements and velocities along the riser are set as zeroes.

\section{Finite Element Model of the Offshore Drilling Riser}

As can be observed in equation (24), it is a challenge to solve the dynamic model of the riser analytically. Under such circumstances, the finite element method is always considered due to its extensive adaptability.
3.1. Discretization of the Dynamic Model. As the first step of finite element analysis, the developed dynamic model of the riser should be transformed into a finite element model. In order to discretize the developed riser model, an approximate solution of the deflection of the $i$ th riser segment is set as

$$
\varpi_{i}(x, t)=[\mathbf{N}(x)]\left\{\mathbf{S}_{i}(t)\right\},
$$

where the Hermite cubic interpolation function for a bending beam element is used as the shape function:

$$
\begin{gathered}
{[\mathbf{N}(x)]=\left[N_{1}(x) N_{2}(x) N_{3}(x) N_{4}(x)\right],} \\
\left\{\begin{array}{l}
N_{1}(x)=1-3 \varepsilon^{2}+2 \varepsilon^{3} \\
N_{2}(x)=\left(\varepsilon-2 \varepsilon^{2}+\varepsilon^{3}\right) l \\
N_{3}(x)=3 \varepsilon^{2}-2 \varepsilon^{3} \\
N_{4}(x)=\left(\varepsilon^{3}-\varepsilon^{2}\right) l \\
\varepsilon=\frac{x-x_{i}}{l}, x \in\left[x_{i} x_{i}+l\right]
\end{array}\right.
\end{gathered}
$$

where $l$ is the length for a riser element.

For the $i$ th riser segment, the nodal displacement vector can be set as

$$
\left\{\mathbf{S}_{i}(t)\right\}=\left(\begin{array}{c}
\omega_{i}(t) \\
\theta_{i}(t) \\
\omega_{i+1}(t) \\
\theta_{i+1}(t)
\end{array}\right)
$$


When introducing the approximate solution shown in equation (27) into the dynamic model of the offshore drilling riser shown in equation (24), the variation of functional equation can be expressed as

$$
\begin{aligned}
& \delta \prod_{P}=\int_{0}^{T_{w}} \int_{0}^{L}\left(\begin{array}{c}
\delta\left(\frac{1}{2}\left(m_{r}+m_{m}\right)[\mathbf{N}(x)]^{2}\left(\frac{\partial\{\mathbf{S}(t)\}}{\partial t}\right)^{2}\right)-\delta\left(\frac{1}{2} E I_{x}\left(\frac{\partial^{2}[\mathbf{N}(x)]}{\partial x^{2}}\right)^{2}\{\mathbf{S}(t)\}^{2}\right. \\
+\frac{1}{2} F_{\text {axial }}(x)\left(\frac{\partial[\mathbf{N}(x)]}{\partial x}\right)^{2}\{\mathbf{S}(t)\}^{2}+\delta\left(F_{f}(x, t) \cdot[\mathbf{N}(x)]\{\mathbf{S}(t)\}\right)
\end{array}\right) \mathrm{d} x \mathrm{~d} t, \\
& =\int_{0}^{T_{w}} \int_{0}^{L}\left(\begin{array}{c}
-\left(m_{r}+m_{m}\right)[\mathbf{N}(x)]^{2} \frac{\partial^{2}\{\mathbf{S}(t)\}}{\partial t^{2}} \cdot \delta\{\mathbf{S}(t)\}-E I_{x}\left(\frac{\partial^{2}[\mathbf{N}(x)]}{\partial x^{2}}\right)^{2}\{\mathbf{S}(t)\} \cdot \delta\{\mathbf{S}(t)\} \\
-F_{\text {axial }}(x)\left(\frac{\partial[\mathbf{N}(x)]}{\partial x}\right)^{2}\{\mathbf{S}(t)\} \cdot \delta\{\mathbf{S}(t)\}+F_{f}(x, t) \cdot[\mathbf{N}(x)] \cdot \delta\{\mathbf{S}(t)\}
\end{array}\right) \mathrm{d} x \mathrm{~d} t, \\
& =0 .
\end{aligned}
$$

Further considering the arbitrary $\delta\{\mathbf{S}(t)\}$, equation (30) can be simplified as

$$
\int_{0}^{T_{w}} \int_{0}^{L}\left(\begin{array}{c}
-\left(m_{r}+m_{m}\right)[\mathbf{N}(x)]^{2} \frac{\partial^{2}\{\mathbf{S}(t)\}}{\partial t^{2}}-E I_{x}\left(\frac{\partial^{2}[\mathbf{N}(x)]}{\partial x^{2}}\right)^{2}\{\mathbf{S}(t)\} \\
-F_{\text {axial }}(x)\left(\frac{\partial[\mathbf{N}(x)]}{\partial x}\right)^{2}\{\mathbf{S}(t)\}+F_{f}(x, t) \cdot[\mathbf{N}(x)]
\end{array}\right) \mathrm{d} x \mathrm{~d} t=0
$$

Subsequently, a series of matrixes for the riser element are set as follows:

$$
\begin{aligned}
{\left[\mathbf{M}_{r m}\right]^{e} } & =\int_{0}^{l}\left(m_{r}+m_{m}\right)[\mathbf{N}(x)]^{T}[\mathbf{N}(x)] \mathrm{d} x, \\
{\left[\mathbf{M}_{f I}\right]^{e} } & =\int_{0}^{l} \frac{1}{4} \pi D^{2} \rho_{w}\left(C_{i}-1\right)[\mathbf{N}(x)]^{T}[\mathbf{N}(x)] \mathrm{d} x, \\
{\left[\mathbf{K}_{E I}\right]^{e} } & =\int_{0}^{l} E I_{x}\left[\frac{\partial^{2}[\mathbf{N}(x)]}{\partial x^{2}}\right]^{T}\left[\frac{\partial^{2}[\mathbf{N}(x)]}{\partial x^{2}}\right] \mathrm{d} x, \\
{\left[\mathbf{K}_{F a}\right]^{e} } & =\int_{0}^{l}\left(F_{\mathrm{top}}-\left(1-f_{b}\right)\left(m_{r}+m_{m}\right) g(L-x)\right)\left[\frac{\partial[\mathbf{N}(x)]}{\partial x}\right]^{T}\left[\frac{\partial[\mathbf{N}(x)]}{\partial x}\right] \mathrm{d} x, \\
{\left[C_{f D}\right]^{e} } & =\int_{0}^{l} \frac{1}{2} C_{d} D \rho_{w} B(x, t)[\mathbf{N}(x)]^{T}[\mathbf{N}(x)] \mathrm{d} x, \\
\{f\}^{e} & =\int_{0}^{l}\left(\frac{1}{2} C_{d} D \rho_{w} B(x, t)\left(u_{w}(x, t)+u_{c}(x)\right)+\frac{1}{4} \pi D^{2} \rho_{w} C_{i} a_{w}(x, t)\right) \cdot[\mathbf{N}(x)]^{T} \mathrm{~d} x,
\end{aligned}
$$


where the integration parts in the abovementioned matrixes can be further calculated by introducing the Hermite cubic interpolation function and its first and second derivatives as

$$
\begin{aligned}
& \int_{0}^{l}[\mathbf{N}(x)]^{T}[\mathbf{N}(x)] d x=\left[\begin{array}{cccc}
\frac{13}{35} l & \frac{11}{210} l^{2} & \frac{9}{70} l & -\frac{13}{420} l^{2} \\
\frac{11}{210} l^{2} & -\frac{1}{105} l^{3} & \frac{13}{420} l^{2} & -\frac{11}{140} l^{3} \\
\frac{9}{70} l & \frac{13}{420} l^{2} & \frac{13}{35} l & -\frac{11}{210} l^{2} \\
-\frac{13}{420} l^{2} & -\frac{11}{140} l^{3} & -\frac{11}{210} l^{2} & \frac{1}{105} l^{3}
\end{array}\right], \\
& \int_{0}^{l}\left[\frac{\partial[\mathbf{N}(x)]}{\partial x}\right]^{T}\left[\frac{\partial[\mathbf{N}(x)]}{\partial x}\right] \mathrm{d} x=\left[\begin{array}{cccc}
\frac{6}{5 l} & \frac{1}{10} & -\frac{6}{5 l} & \frac{1}{10} \\
\frac{1}{10} & \frac{2}{15} l & -\frac{1}{10} & -\frac{1}{30} l \\
-\frac{6}{5 l} & -\frac{1}{10} & \frac{6}{5 l} & -\frac{1}{10} \\
\frac{1}{10} & -\frac{1}{30} l & -\frac{1}{10} & \frac{2}{15} l
\end{array}\right] \\
& \int_{0}^{l}\left[\frac{\partial^{2}[\mathbf{N}(x)]}{\partial x^{2}}\right]^{T}\left[\frac{\partial^{2}[\mathbf{N}(x)]}{\partial x^{2}}\right] \mathrm{d} x=\left[\begin{array}{cccc}
\frac{12}{l^{3}} & \frac{6}{l^{2}} & -\frac{12}{l^{3}} & \frac{6}{l^{2}} \\
\frac{6}{l^{2}} & \frac{4}{l} & -\frac{6}{l^{2}} & \frac{2}{l} \\
-\frac{12}{l^{3}} & -\frac{6}{l^{2}} & \frac{12}{l^{3}} & -\frac{6}{l^{2}} \\
\frac{6}{l^{2}} & \frac{2}{l} & -\frac{6}{l^{2}} & \frac{4}{l}
\end{array}\right] .
\end{aligned}
$$

Finally, the integrated finite element model for the riser system can be written in a compact form as

$$
\left(\left[\mathbf{M}_{r m}\right]+\left[\mathbf{M}_{f I}\right]\right)\{\ddot{S}\}+\left[\mathbf{C}_{f D}\right]\{\dot{S}\}+\left(\left[\mathbf{K}_{E I}\right]+\left[\mathbf{K}_{F a}\right]\right)\{\mathbf{S}\}=\{\mathbf{f}\},
$$

where

$$
\begin{gathered}
\{\dot{S}\}=\frac{\partial\{\mathbf{S}(t)\}}{\partial t}, \\
\{\ddot{S}\}=\frac{\partial^{2}\{\mathbf{S}(t)\}}{\partial t^{2}} .
\end{gathered}
$$

3.2. Numerical Solution of the Finite Element Model. The Newmark integration algorithm is applied to solve the developed finite element model, and thus, the dynamic responses of the riser system in time domain can be obtained via numerical simulation. Specifically, two basic parameters for Newmark integration algorithm are set as $\alpha=0.25$ and $\delta=0.5$. Thus, other eight integration constants are described

$$
\left\{\begin{array}{l}
a_{0}=\frac{1}{\left(\alpha^{*} \mathrm{~d} t\right)^{2}}, \\
a_{1}=\frac{\delta}{\left(\alpha^{*} \mathrm{~d} t\right)}, \\
a_{2}=\frac{1}{\left(\alpha^{*} \mathrm{~d} t\right)}, \\
a_{3}=\frac{1}{(2 a)-1}, \\
a_{4}=\frac{\delta}{\alpha-1} \\
a_{5}=\left(\frac{\delta}{\alpha-2}\right)^{*} \mathrm{~d} t \\
a_{6}=(1-\delta)^{*} \mathrm{~d} t \\
a_{7}=\delta * \mathrm{~d} t,
\end{array}\right.
$$

where $d t$ is the size of time step. 


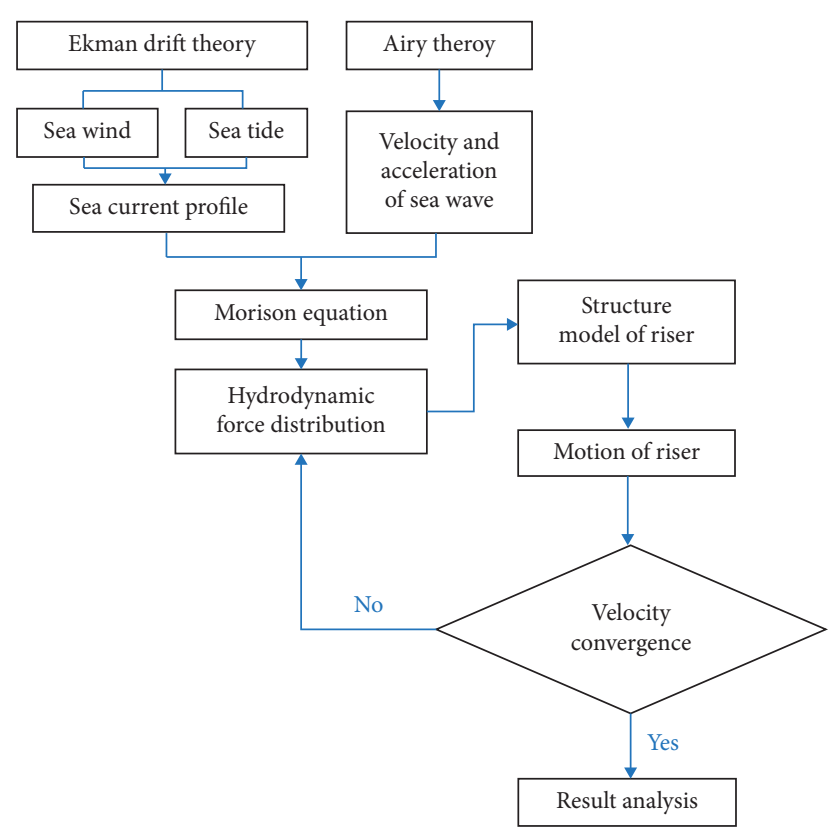

FIgURE 2: Flow chart for the solution procedure via an iterative calculation.

In particular, according to both the displacement vector $\left\{\mathbf{S}_{0}\right\}$ and the velocity vector $\left\{\dot{S}_{0}\right\}$ in the initial time, the initial acceleration vector can be calculated, firstly, as

$$
\begin{aligned}
\left\{\ddot{S}_{0}\right\}= & \left(\left[\mathbf{M}_{r m}\right]+\left[\mathbf{M}_{f I}\right]\right)^{-1}\left(\{\mathbf{f}\}_{i}-\left[\mathbf{C}_{f D}\right]\left\{\dot{S}_{0}\right\}\right. \\
& \left.+\left(\left[\mathbf{K}_{E I}\right]+\left[\mathbf{K}_{F a}\right]\right)\left\{\mathbf{S}_{0}\right\}\right) .
\end{aligned}
$$

During the following numerical integration using Newmark algorithm, the effective stiffness is calculated as

$$
\mathbf{K}_{e}=\left(\left[\mathbf{K}_{E I}\right]+\left[\mathbf{K}_{F a}\right]\right)+a_{0} \cdot\left(\left[\mathbf{M}_{r m}\right]+\left[\mathbf{M}_{f I}\right]\right)+a_{1} \cdot\left[\mathbf{C}_{f D}\right] .
$$

Then, the displacement vector for the $(i+1)$ th time can be calculated based on the corresponding results obtained from the $i$ th time:

$$
\left\{\mathbf{S}_{i+1}\right\}=\left(\mathbf{K}_{e}\right)^{-1}\left(\left\{\mathbf{f}_{i+1}\right\}+\left(\left[\mathbf{M}_{r m}\right]+\left[\mathbf{M}_{f I}\right]\right) B_{1}+\left[\mathbf{C}_{f D}\right] B_{2}\right),
$$

where

$$
\left\{\begin{array}{l}
B_{1}=a_{0}\left\{\mathbf{S}_{i}\right\}+a_{2}\left\{\dot{S}_{i}\right\}+a_{3}\left\{\ddot{S}_{i}\right\}, \\
B_{2}=a_{1}\left\{\mathbf{S}_{i}\right\}+a_{4}\left\{\dot{S}_{i}\right\}+a_{5}\left\{\ddot{S}_{i}\right\} .
\end{array}\right.
$$

Subsequently, the acceleration vector and velocity vector for the $(i+1)$ th time can be calculated in sequence as

$$
\begin{aligned}
& \left\{\ddot{S}_{i+1}\right\}=a_{0}\left(\left\{\mathbf{S}_{i+1}\right\}-\left\{\mathbf{S}_{i}\right\}\right)-a_{2}\left\{\dot{S}_{i}\right\}-a_{3}\left\{\ddot{S}_{i}\right\}, \\
& \left\{\dot{S}_{i+1}\right\}=\left\{\dot{S}_{i}\right\}+a_{6}\left\{\ddot{S}_{i}\right\}+a_{7}\left\{\ddot{S}_{i+1}\right\} .
\end{aligned}
$$

In addition, during the numerical simulation, both the damping matrix $\left[C_{f D}\right]^{e}$ and the vector of external force $\{\mathbf{f}\}^{e}$ depend on the linear factor $B(x, t)$, which is further determined by the velocity of the riser; hence, in order to solve this dynamic model, the iterative procedure is inevitable, and the completed flow chart for the solution procedure is shown in Figure 2.

\section{Dynamic Analyses of Offshore Drilling Risers}

Based on both the finite element model and the corresponding solution procedure developed in Section 3, the dynamic analyses of offshore drilling risers can be carried out comprehensively in this section. In particular, by considering the limitation of the calculation capacity of hardware, meanwhile, according to the test of the grid independence verification, the length of a riser element is set as $5 \mathrm{~m}$, while the time step is set as $0.0005 \mathrm{~s}$. Under such circumstances, for instance, the numerical simulation of a riser with $2000 \mathrm{~m}$ needs to run over 46 hours until this riser system reaches its dynamic stable condition.

4.1. Characteristic Analyses of Dynamic Responses. A series of dynamic responses of the offshore drilling risers with varied parameters are compared according to the vibration and deflection of the riser. The basic parameters for the following numerical simulations are listed in Table 1.

By using the basic parameters listed in Table 1, the ocean environmental loads can be determined firstly. As shown in Figure 3, for a study case whose sea depth is $2000 \mathrm{~m}$, the influence of sea wave concentrates on the region close to the sea surface, and it varies periodically, see subplot (a) and (b), while the velocity distribution of sea current is independent from time, see subplot (c). 
TABLE 1: System parameters for the dynamic analyses of the riser model.

\begin{tabular}{lcc}
\hline Parameter & Symbol & Value \\
\hline Density of the riser & $\rho_{r}$ & $7850 \mathrm{~kg} / \mathrm{m}^{3}$ \\
Elastic modulus & $E$ & $210 \mathrm{GPa}$ \\
External diameter & $D_{r}$ & $0.5334 \mathrm{~m}$ \\
Internal diameter & $d_{r}$ & $0.4826 \mathrm{~m}$ \\
Top tension ratio & $R_{t o p}$ & 1.3 \\
Buoyancy factor & $f_{b}$ & 0.9 \\
Density of water & $\rho_{w}$ & $1050 \mathrm{~kg} / \mathrm{m}^{3}$ \\
Drag coefficient & $C_{d}$ & 0.7 \\
Inertial coefficient & $C_{i}$ & 2.0 \\
Wave amplitude & $h_{w}$ & $6.5 \mathrm{~m}$ \\
Wave period & $T_{w}$ & $8 \mathrm{~s}$ \\
Wind speed & $v_{w}$ & $5 \mathrm{~m} / \mathrm{s}$ \\
Factor of wind speed & $K_{w}$ & 0.03 \\
Strong tidal range & $R_{M}$ & $20 \mathrm{~m}$ \\
Normal tidal range & $R_{d}$ & $1 \mathrm{~m}$ \\
Geographic latitude & $\varphi^{\circ}$ & $10^{\circ} \mathrm{N}$ \\
Density of drilling mud & $\rho_{m}$ & $1200 \mathrm{~kg} / \mathrm{m}^{3}$ \\
Rotational stiffness of the upper joint & $K_{t}$ & $1.1 e 8 \mathrm{~N} \cdot \mathrm{m} / \mathrm{rad}$ \\
Rotational stiffness of the bottom joint & $K_{b}$ & $1.1 e 8 \mathrm{~N} \cdot \mathrm{m} / \mathrm{rad}$ \\
\hline
\end{tabular}

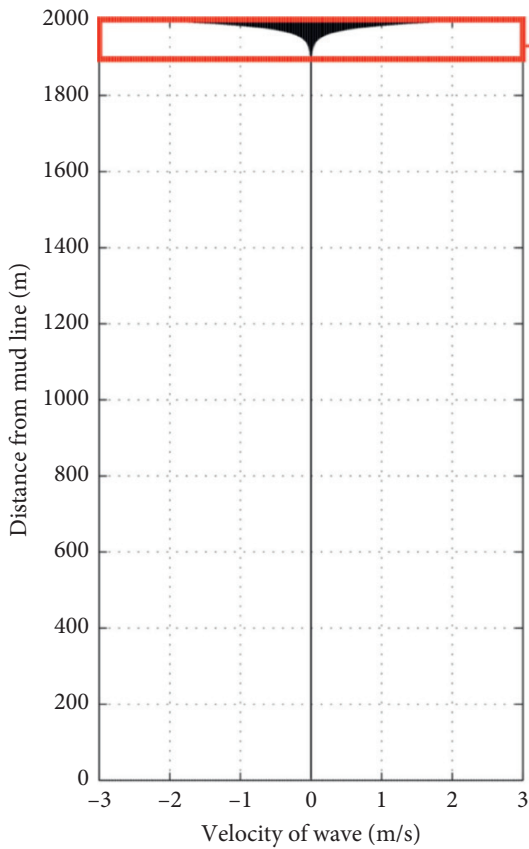

(a)

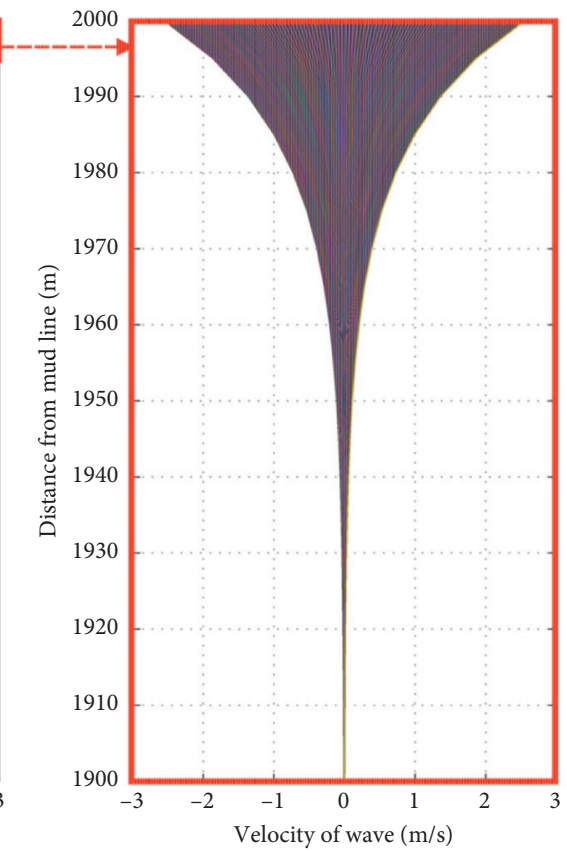

(b)

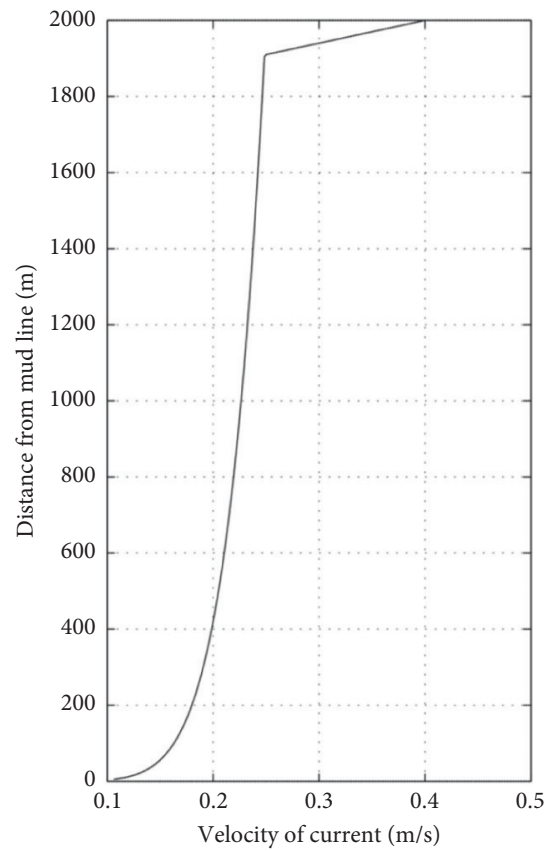

(c)

Figure 3: Ocean environmental loads, (a) distribution of wave velocity, (b) enlarged view of the wave velocity distribution close to the ocean surface, (c) distribution of sea current velocity.

Under the excitation of the ocean environmental loads shown in Figure 3, the obtained dynamic responses of the offshore drilling riser are shown in Figure 4. During the numerically simulated 300 seconds, the offshore drilling riser experienced a fast lateral deflection in the first two wave periods, namely, $0 \sim 16 \mathrm{~s}$, and then, encountered a reciprocating deflection phase which lasted for a long time, $17 \mathrm{~s} \sim 260 \mathrm{~s}$; finally, the riser reached its dynamic stable condition, i.e., periodic oscillation, in $261 s \sim 300$ s, see the curves coloured by red in Figure 4(a)-4(c).
In such the three phases, the vibration trajectories of the riser in every $200 \mathrm{~m}$ depth are displayed in Figure 4(d)-4(f), respectively, which further display the characteristics of dynamic responses of an offshore drilling riser.

The dynamic stable condition of the riser is expected to be maintained if the ocean environmental loads are not changed, and the corresponding dynamic responses of the riser are further shown in Figure 5. In addition to the distribution of lateral deflection of the riser in subplot (a), 


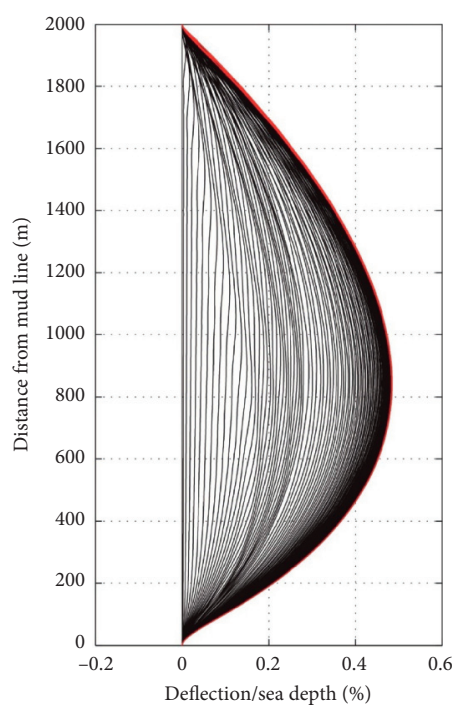

(a)

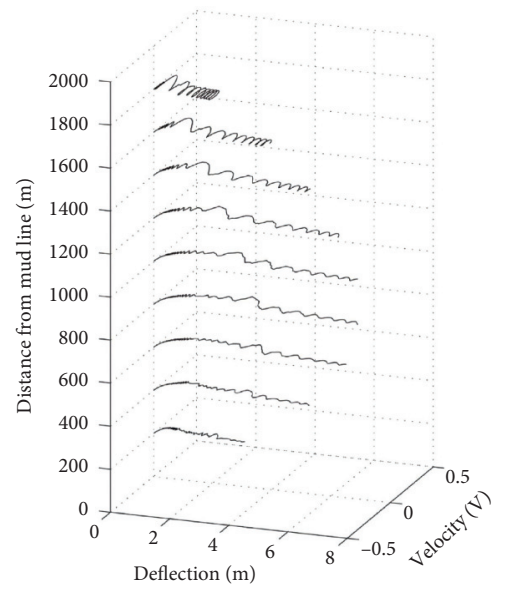

(d)

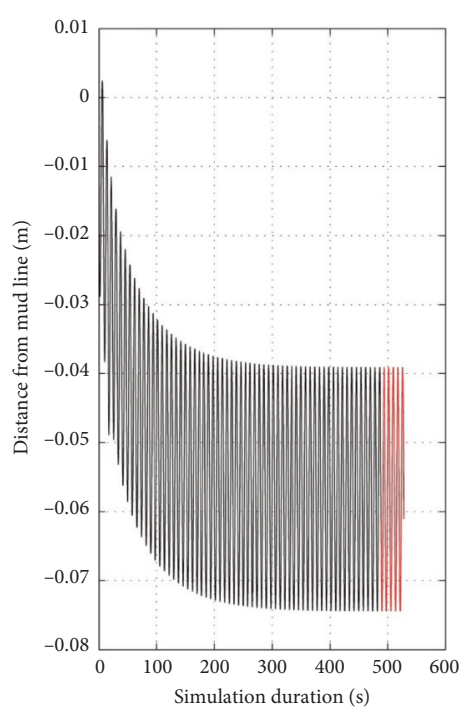

(b)

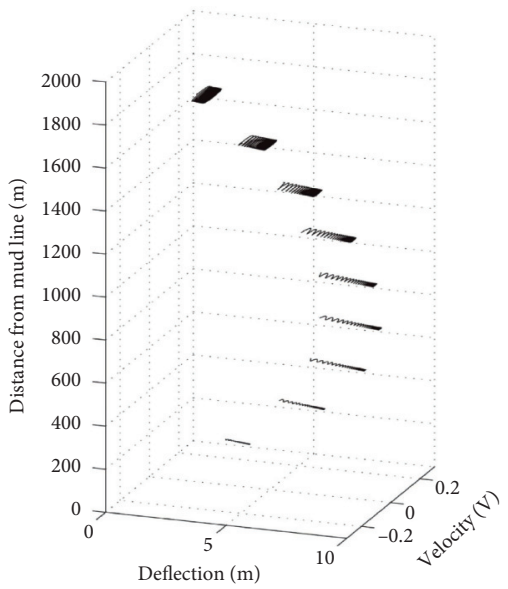

(e)

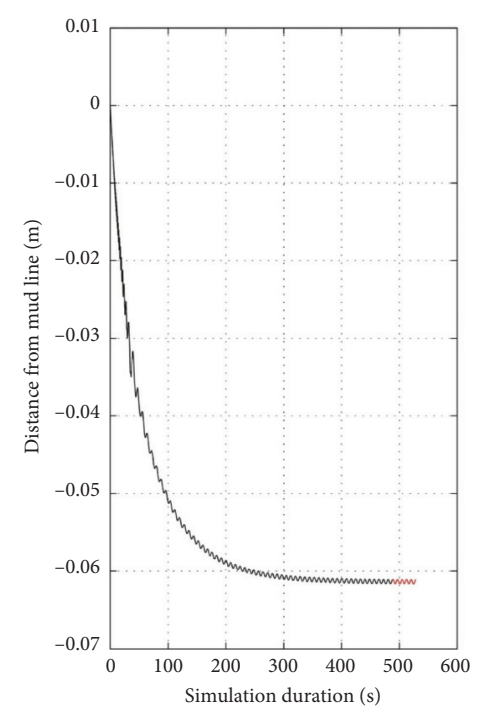

(c)

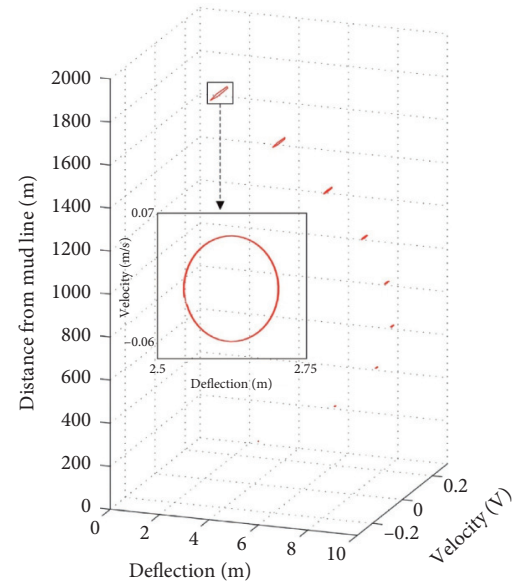

(f)

Figure 4: Dynamic responses for an offshore drilling riser with $2000 \mathrm{~m}$ during the numerical simulation in $0 \sim 300 \mathrm{~s}$, (a) distribution of lateral deflection, (b) time history of deflection angle of the upper joint, and (c) that of the bottom joint. The vibration trajectories of the riser in every $200 \mathrm{~m}$ depth are displayed for (d) fast lateral deflection phase, (e) reciprocating deflection phase, and (f) dynamic stable condition phase, respectively.

the distributions of both the bending stress (b) and the deflection angle of the riser (c) are also displayed, which are calculated as follows:

$$
\begin{aligned}
\delta(x, t) & =\frac{D_{r} M(x, t)}{2 I_{x}}, \\
\theta_{a}(x, t) & =\frac{180 \cdot \theta(x, t)}{\pi} .
\end{aligned}
$$

As can be seen in Figure 5, the maximal lateral deflection appears in the middle part of the riser, and both the maximal bending stress and the largest deflection angle of the riser are observed in the positions close to both the top and bottom ends of the riser.
4.2. Analyses of Influences of Key Parameters. Primarily, the influence of riser length, namely, sea depth, is considered, and three different study cases, including the risers with the length of $500 \mathrm{~m}, 1000 \mathrm{~m}$, and $1500 \mathrm{~m}$, are compared. Moreover, in all the studied cases, the risers have reached their dynamic stable conditions since only the numerical results for the last $40 \mathrm{~s}(261 \mathrm{~s} \sim 300 \mathrm{~s})$ in the total $300 \mathrm{~s}$ simulation duration were analysed. As the results displayed in Figure 6, the riser system working in shallow water has stronger vibration, since both its vibration amplitude and bending stress are larger than other risers working in deeper water, see the blue curves in both the subplots (a) and (b). However, both the lateral deflection and the deflection angle of a longer riser are relatively larger than the short ones, see the red curves in both the subplots (a) and (c). 


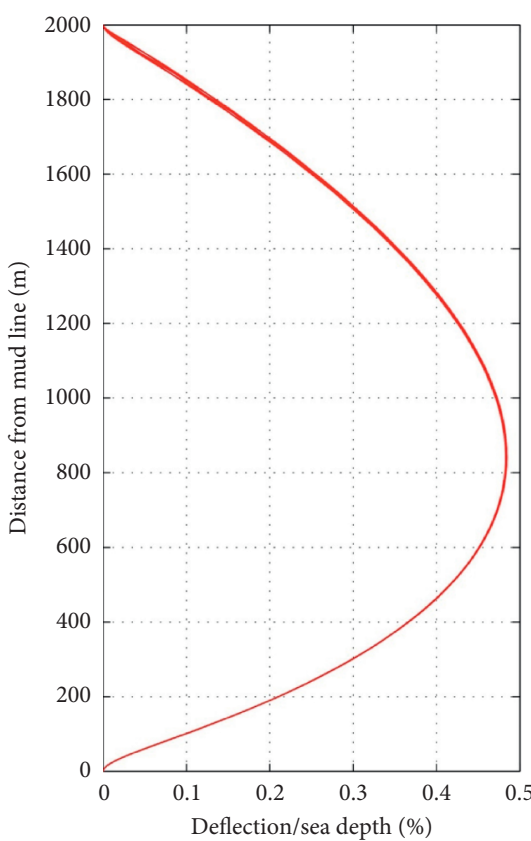

(a)

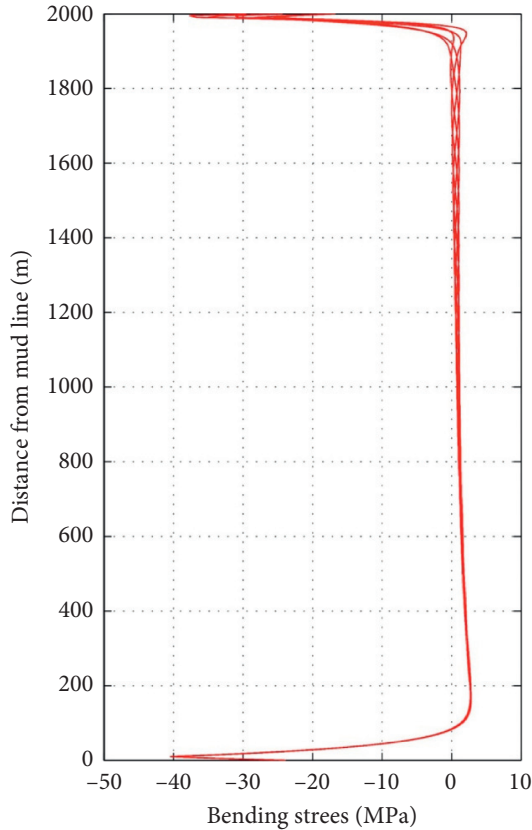

(b)

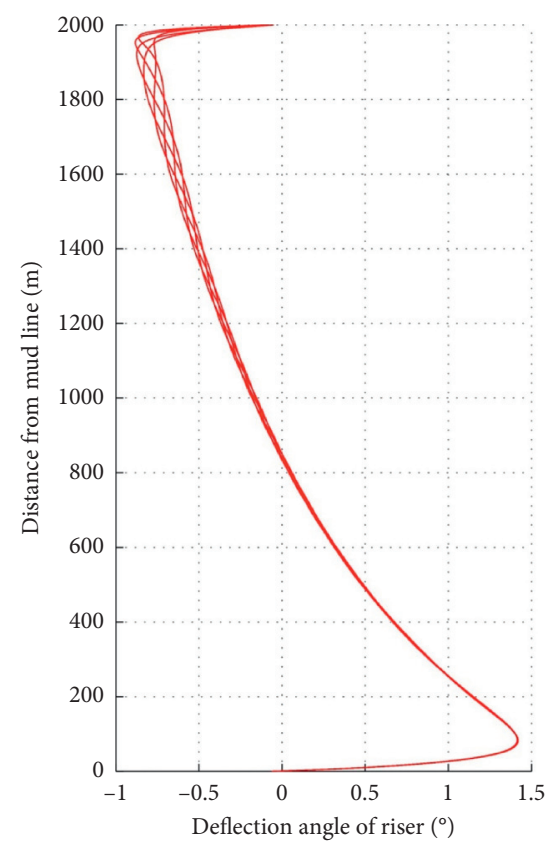

(c)

Figure 5: Dynamic responses for a riser with $2000 \mathrm{~m}$ in its dynamic stable condition, (a) distribution of lateral deflection, (b) distribution of bending stress, and (c) distribution of deflection angle of the riser.

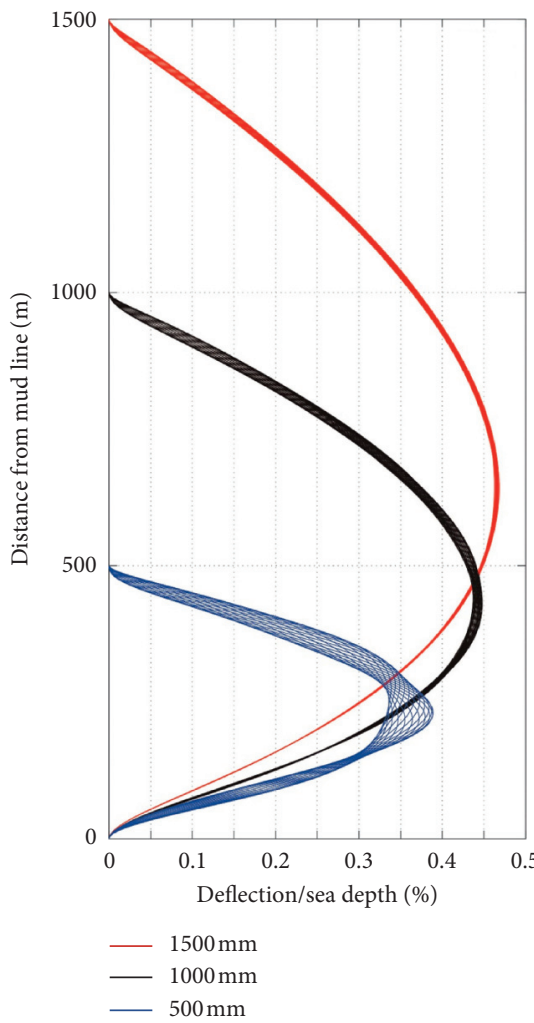

(a)

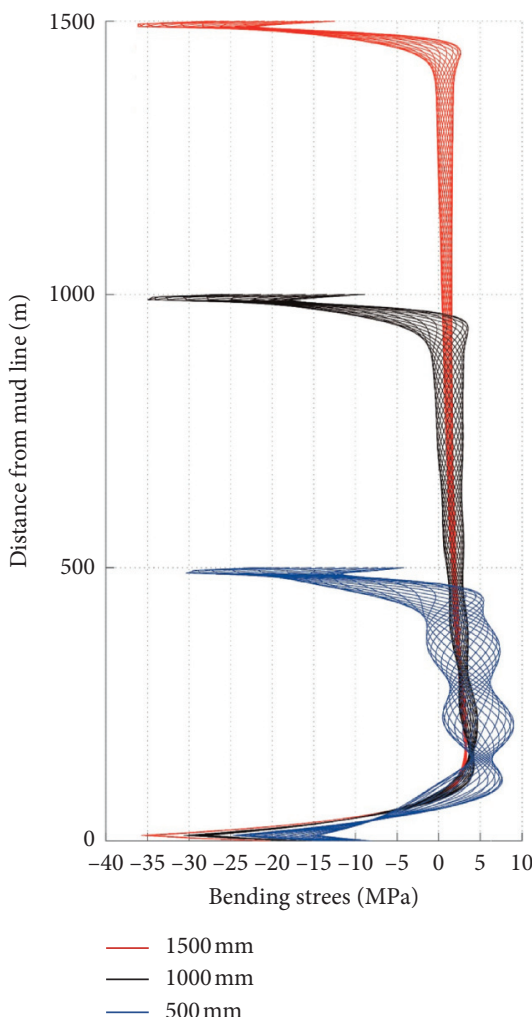

(b)

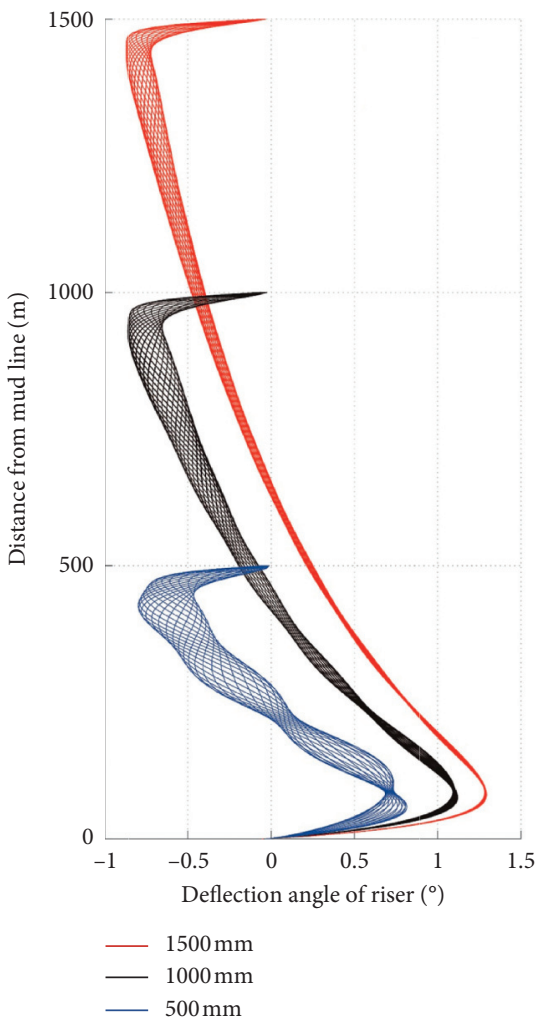

(c)

FIGURE 6: Comparison of dynamic responses for risers with different lengths, (a) distribution of lateral deflection, (b) distribution of bending stress, and (c) distribution of deflection angle of the riser. 


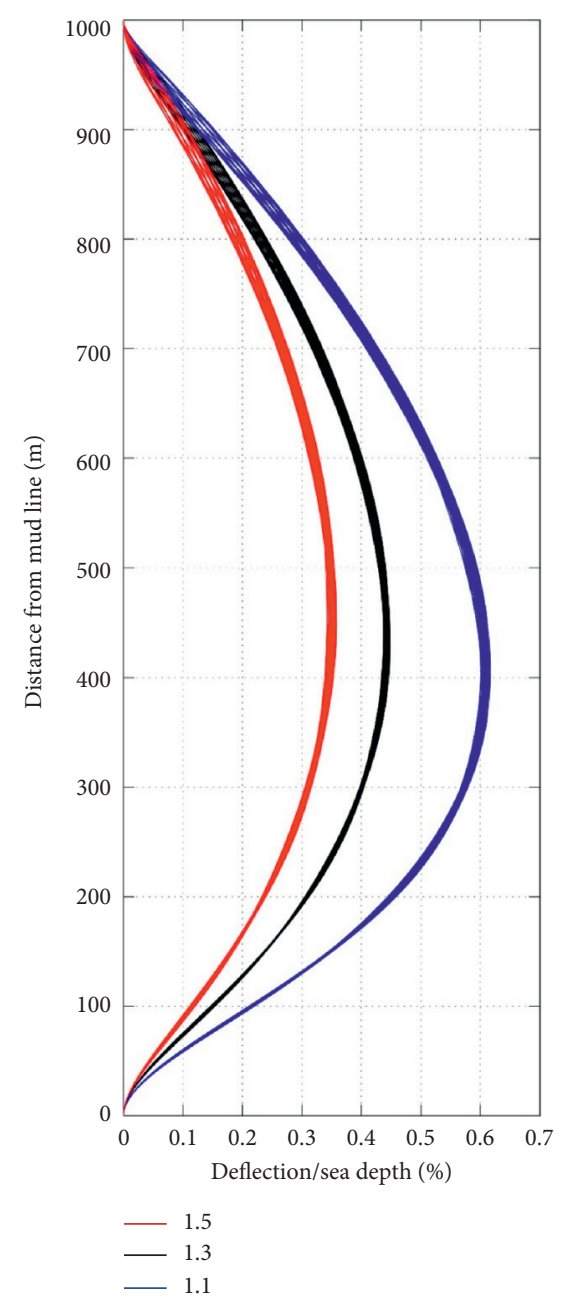

(a)

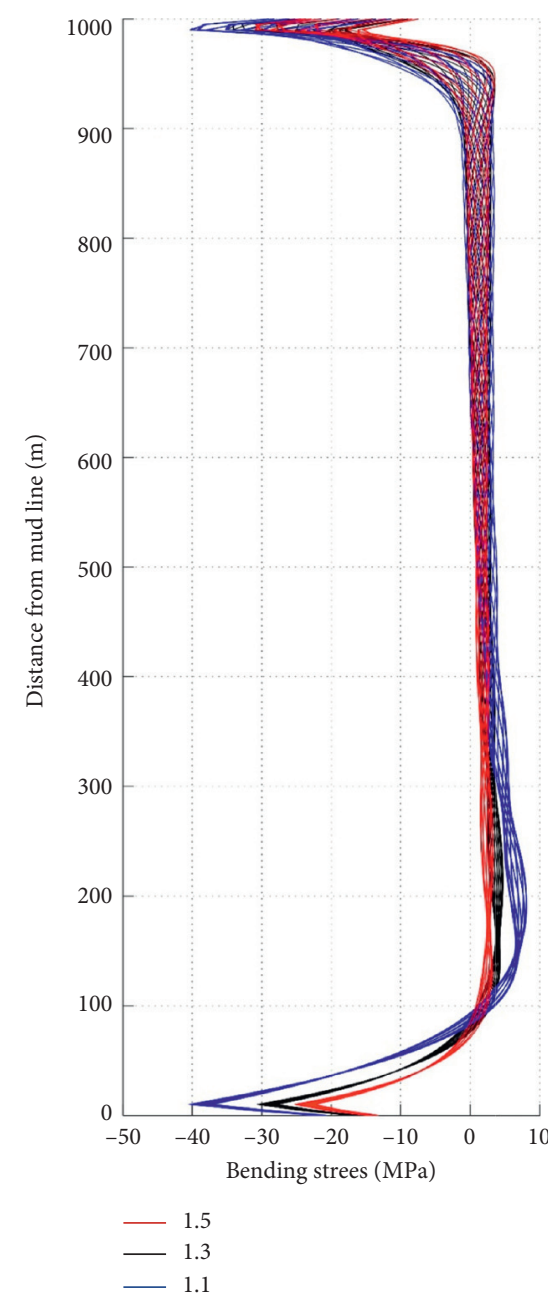

(b)

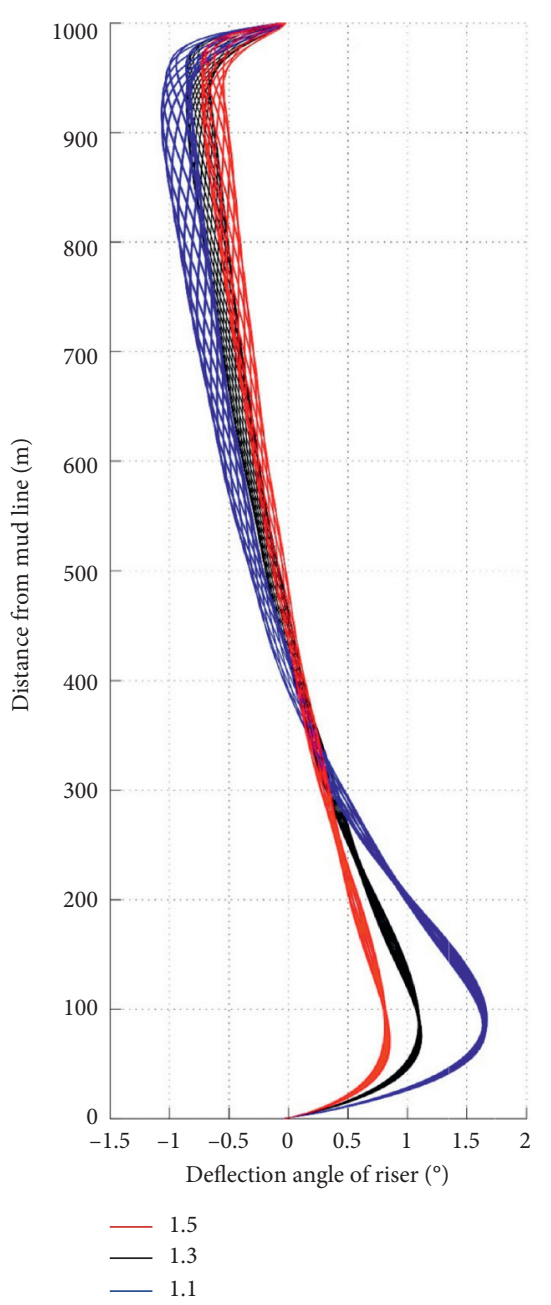

(c)

FIGURE 7: Comparison of dynamic responses for risers with different top tension ratios, (a) distribution of lateral deflection, (b) distribution of bending stress, and (c) distribution of deflection angle of the riser.

Subsequently, the influence of the top tension is analysed, and also, three different study cases, including the ratio of top tension for 1.1, 1.3, and 1.5, are compared. As the results shown in Figure 7, obviously, once a higher top tension is applied, both the vibration and the lateral deflection of the riser can be effectively controlled, see the red curves in subplot (a). Furthermore, when the high top tension is applied in the riser system, both the bending stress and the deflection angle along the riser can also be decreased, see the red curves in both the subplots (b) and (c).

Finally, the influence of the buoyant factor is discussed. The buoyant factor is mainly determined by the design strategy of the buoyancy blocks which parcel the riser outside. Also, three different study cases, including the buoyant factor for $0.5,0.7$, and 0.9 , are compared. As the results shown in Figure 8, when more buoyancy blocks are added to parcel the riser to decrease the requirement of the lifting capacity of the drilling platform or vessel, the lateral deflection of the riser is obviously increased, see the black curves in subplot (a); meanwhile, the maximal bending stress and the maximal deflection angle of riser are also increased, see the black curves in both the subplots (b) and (c). Therefore, in order to control the deflection of the riser, the buoyant factor is suggested to be set small if the lifting capacity of the drilling platform or vessel is strong enough to provide the sufficient top tension. 


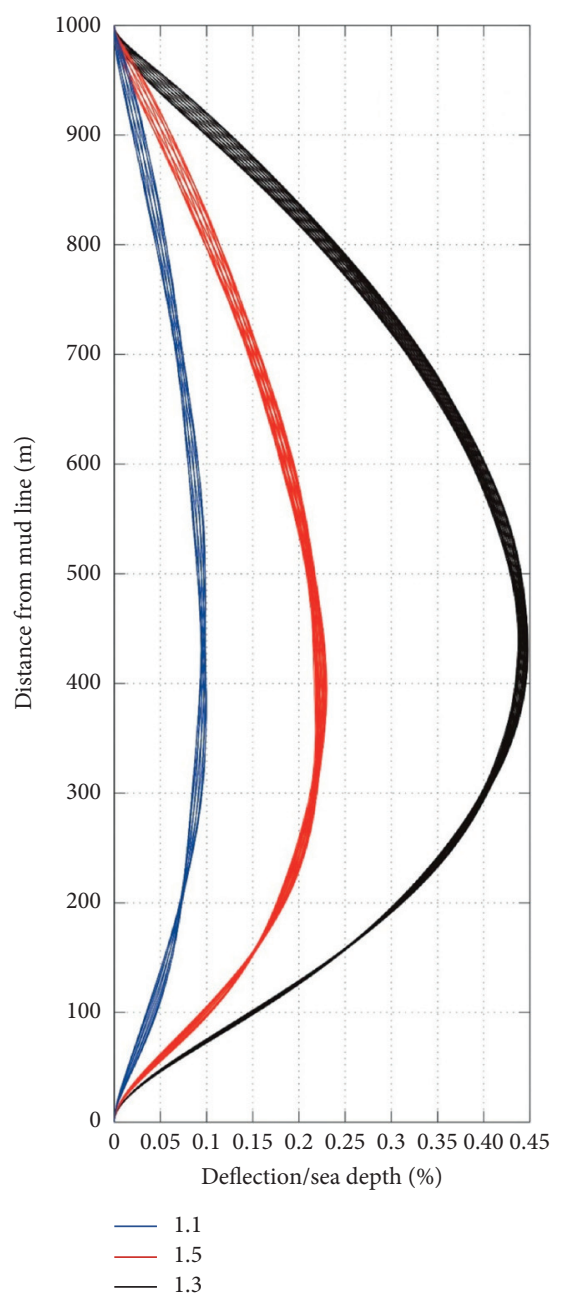

(a)

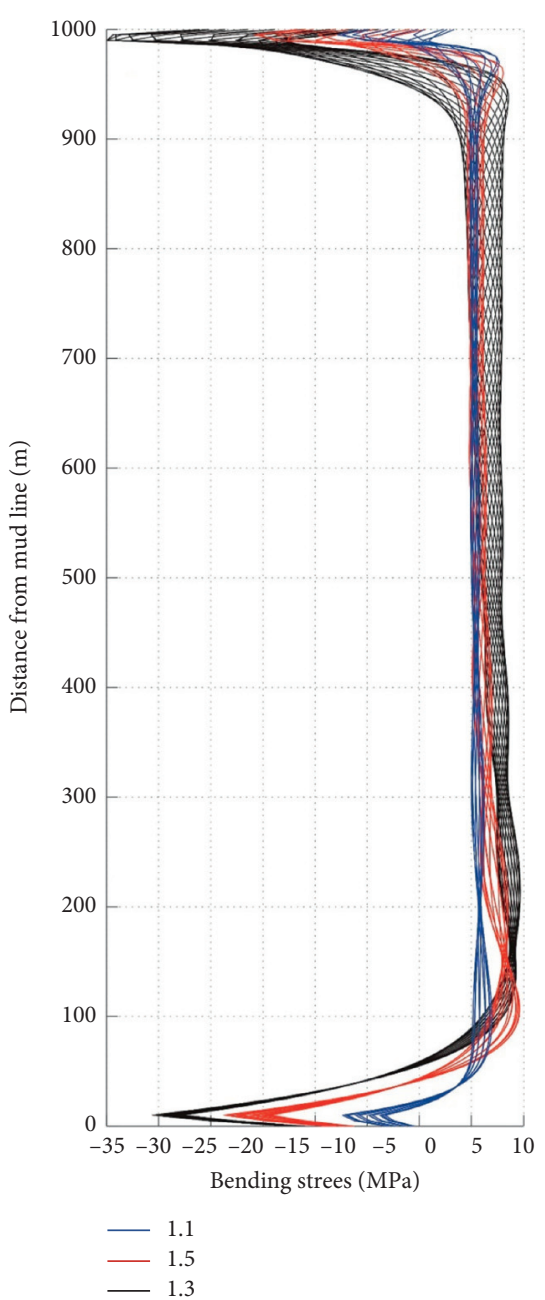

(b)

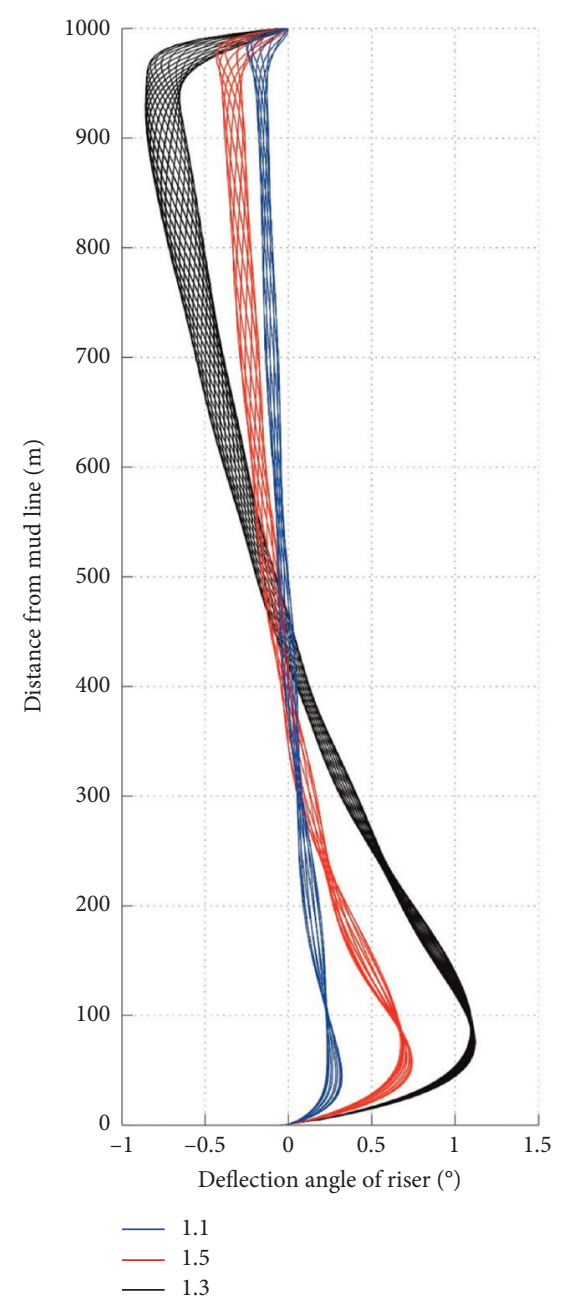

(c)

Figure 8: Comparison of dynamic responses for risers with different buoyant factors, (a) distribution of lateral deflection, (b) distribution of bending stress, and (c) distribution of deflection angle of the riser.

\section{Concluding Remarks}

(1) In this paper, the mathematical model of an offshore drilling riser system developed by using the Hamilton principle was introduced in detail, and the corresponding finite element model was, then, derived, so that the dynamic responses of offshore drilling risers with varied system parameters were numerically simulated by using the standard Newmark integration.

(2) According to the results of numerical simulation, under the influences of variety of ocean environmental loads, an offshore drilling riser will experience a fast lateral deflection phase in the beginning and encounter a reciprocating deflection phase in the following long simulation duration and, then, enter into a periodic oscillation phase when it reaches the dynamic stable condition.

(3) Three key system parameters for an offshore drilling riser were discussed. Specifically, when considering the influence of sea depth, the riser system working in shallow water has severer vibration than that working in deeper water; however, the lateral deflection for a longer riser is relatively larger. In addition, when taking both the top tension and buoyant factor into considerations to control the dynamic responses of a riser, the combination of a higher top tension ratio and a lower buoyant factor was illustrated to be better to fulfill lower vibration and smaller deflection of the riser.

(4) As mentioned in the introduction, the present work in this paper is a start for the authors' whole research plan for analyses of the double nonlinearities of a drilling tube system with pipe-in-pipe structure, including the geometric nonlinearity from the configuration of the drilling tube system, and the contact nonlinearity caused by the interactions between drill string and riser/well. The final target is to precisely predict the dynamic responses of a riserwell-drilling string coupling system when it is applied in deep water drilling. 


\section{Data Availability}

The data used to support the findings of this study are included within the article.

\section{Conflicts of Interest}

The authors declare that they have no conflicts of interest.

\section{Acknowledgments}

This work was supported by the National Natural Science Foundation of China (No. 51904018) and Fundamental Research Funds for the Central Universities (No. FRF-TP18-054A1).

\section{References}

[1] B. G. Burke, "An analysis of marine risers for deep water," in Proceedings of the Offshore Technology Conference, Houston, Texas, USA, May 1973.

[2] M. H. Patel, S. Sarohia, and K. F. Ng, "Finite-element analysis of the marine riser," Engineering Structures, vol. 6, no. 3, pp. 175-184, 1984.

[3] B. Yue, K. C. Man, and D. Walters, "Tension and expansion analysis of pipe-in-pipe risers: Part B finite element modelling," in Proceedings of the International Offshore and Polar Engineering, Anchorage, AK, USA, June 2013.

[4] M. M. Bernitsas, J. E. Kokarakis, and A. Imron, "Large deformation three-dimensional static analysis of deep water marine risers," Applied Ocean Research, vol. 7, no. 4, pp. 178-187, 1985.

[5] Y. Bae and M. M. Bernitsas, "Importance of nonlinearities in static and dynamic analyses of marine risers," in Proceedings of the International Offshore and Polar Engineering Conference, Hague, Netherlands, June1995.

[6] M. Yazdchi and M. A. Crisfeld, "Nonlinear dynamic behaviour of flexible marine pipes and risers," International Journal for Numerical Methods in Engineering, vol. 54, no. 9, pp. 1265-1308, 2002.

[7] W. R. Nair and R. E. Baddour, "Three-dimensional dynamics of a flexible marine riser undergoing large elastic deformations," Multibody System Dynamics, vol. 10, pp. 393-423, 2003.

[8] H.-z. Yang, H. Jiang, Q. Yang, and J.-h. Ding, "Coupled element modeling scheme for the global dynamic analysis of unbonded flexible risers," Journal of Shanghai Jiaotong University (Science), vol. 20, no. 2, pp. 234-242, 2015.

[9] Y. Wang, D. Gao, and J. Fang, "Coupled dynamic analysis of deepwater drilling riser under combined forcing and parametric excitation," Journal of Natural Gas Science and Engineering, vol. 27, pp. 1739-1747, 2015.

[10] L. Mao, Q. Liu, S. Zhou, W. Jiang, Z. Liu, and T. Peng, "Vortex-induced vibration mechanism of drilling riser under shear flow," Petroleum Exploration and Development, vol. 42, no. 1, pp. 112-118, 2015.

[11] L. Mao, Q. Liu, S. Zhou, G. Wang, and Q. Fu, "Deep water drilling riser mechanical behavior analysis considering actual riser string configuration," Journal of Natural Gas Science and Engineering, vol. 33, pp. 240-254, 2016.

[12] P. Wu, Y. Zhang, S. Pang et al., "Nonlinear vibration analysis of deepwater top tension riser under drilling condition,"
Journal of Dynamics and Control Physical Review Letters, vol. 17, no. 2, pp. 112-120, 2019.

[13] H. Ren, M. Zhang, J. Cheng et al., "Magnification of hydrodynamic coefficients on a flexible pipe fitted with helical strakes in oscillatory flows," Ocean Engineering, vol. 210, Article ID 107543, 2020.

[14] C. Liu, S. Fu, M. Zhang, H. Ren, and Y. Xu, "Hydrodynamics of a flexible cylinder under modulated vortex-induced vibrations," Journal of Fluids and Structures, vol. 94, Article ID 102913, 2020.

[15] Y. Chang, G. Chen, and L. Xu, "Influential factors for the design of ultra-deepwater drilling risers," Petroleum Exploration and Development, vol. 36, no. 4, pp. 523-528, 2009.

[16] S. S. Ge, W. He, B. V. E. How, and Y. S. Choo, "Boundary control of a coupled nonlinear flexible marine riser," IEEE Transactions on Control Systems Technology, vol. 18, no. 5, pp. 1080-1091, 2010.

[17] Y. Wang, D. Gao, and J. Fang, "Optimization analysis of the riser top tension force in deepwater drilling: Aiming at the minimum variance of lower flexible joint deflection angle," Journal of Petroleum Science and Engineering, vol. 146, pp. 149-157, 2016.

[18] M. Liao, Y. Zhou, Y. Su, Z. Lian, and H. Jiang, "Dynamic analysis and multi-objective optimization of an offshore drilling tube system with pipe-in-pipe structure," Applied Ocean Research, vol. 75, pp. 85-99, 2018.

[19] F. Guo, Y. Liu, F. Luo, and Y. Wu, "Vibration suppression and output constraint of a variable length drilling riser system," Journal of the Franklin Institute, vol. 356, no. 3, pp. 1177-1195, Article ID 106619, 2019.

[20] X. Liu, X. Wang, Y. Jiang, G. Chen, L. Xu, and C. Li, “An optimization method for the suppression device configuration of risers," Ocean Engineering, vol. 194, pp. 1177-1195, Article ID 106619, 2019.

[21] Y. Wu, N. Sun, H. Chen, and Y. Fang, "Adaptive output feedback control for 5-DOF varying-cable-length tower cranes with cargo mass estimation," IEEE Transactions on Industrial Informatics, p. 1, 2020.

[22] T. Yang, N. Sun, H. Chen et al., "Observer-based nonlinear control for tower cranes suffering from uncertain friction and actuator constraints with experimental verification," IEEE Transactions on Industrial Electronics, p. 1, 2020.

[23] O. M. Faltinsen, Sea Loads on Ships and Offshore Structures, Cambridge University Press, Cambridge, England, 1998. 